\title{
هำ Volumetric brain analysis in neurosurgery: Part 1. Particle filter segmentation of brain and cerebrospinal fluid growth dynamics from MRI and CT images
}

\author{
Jason G. Mandell, MS, MD, ${ }^{1,2}$ Jack W. Langelaan, PhD, ${ }^{3}$ Andrew G. Webb, PhD, ${ }^{4}$ \\ and Steven J. Schiff, MD, PhD ${ }^{1,5,6}$
}

${ }^{1}$ Center for Neural Engineering, Department of Engineering Science and Mechanics, and Departments of ${ }^{2}$ Bioengineering, ${ }^{3}$ Aerospace Engineering, ${ }^{5}$ Neurosurgery, and ${ }^{6}$ Physics, The Pennsylvania State University, University Park, Pennsylvania; and ${ }^{4}$ C.J. Gorter Center for High Field MRI, Department of Radiology, Leiden University Medical Center, Leiden, The Netherlands

\begin{abstract}
OBJECT Accurate edge tracing segmentation remains an incompletely solved problem in brain image analysis. The authors propose a novel algorithm using a particle filter to follow the boundary of the brain in the style often used in autonomous air and ground vehicle navigation. Their goals were to create a versatile tool to segment brain and fluid in $\mathrm{MRI}$ and CT images of the developing brain, lay the foundation for an intelligent automated edge tracker that is modality independent, and segment normative data from MRI that can be applied to both MRI and CT.
\end{abstract}

METHODS Simulated MRI data sets were used to train and evaluate the particle filter segmentation algorithm. The method was then applied to produce normative growth curves for children and adolescents from 0 to 18 years of age for brain and fluid from MR images from the National Institutes of Health pediatric database and these data were compared to historical results. The authors further adapted this method for use with CT images of pediatric hydrocephalus and compared the results with hand-segmented data.

RESULTS Segmentation of simulated MRI data with varied levels of noise $(0 \%-9 \%)$ and spatial inhomogeneity $(0 \%$ $40 \%$ ) resulted in percent errors ranging from $0.06 \%$ to $5.38 \%$ for brain volume and $2.45 \%$ to $22.3 \%$ for fluid volume. The authors used this tool to create normal brain and CSF growth curves from MR images. The calculated growth curves showed excellent consistency with historical data. Additionally, compared with manual segmentation the particle filter accurately segmented brain and fluid volumes from CT scans of 5 pediatric patients with hydrocephalus $(p<0.001)$.

CONCLUSIONS The authors have produced the first normative brain and CSF growth curves for children and adolescents $0-18$ years of age. In addition, this study includes the first use of a particle filter as an edge tracker in image segmentation and offers a semiautomatic method to segment both pediatric and adult brain data from MR and CT images. The particle filter has the potential to be further automated toward a clinical rather than research tool with both of these modalities. Because of its modality independence, it has the capability to allow CT to be a more effective diagnostic tool for neurological disorders, a task of substantial importance in emergency settings and in developing countries where CT is often the only available method of brain imaging.

http://thejns.org/doi/abs/10.3171/2014.9.PEDS12426

KEY WORDS segmentation; particle filter; brain growth; cerebrospinal fluid; hydrocephalus; technique

$\mathrm{S}$ EGMENTATION in image analysis is the partitioning of a digital image into non-overlapping regions that are homogeneous with respect to some characteristic, such as intensity. ${ }^{21}$ In neurosurgery there is a long history of applying segmentation tools, and in particular volumetric methods, to the evaluation of patients with hydrocephalus $^{19}$ and candidates for epilepsy surgery. ${ }^{5}$ Current research on automated brain segmentation is largely di- rected toward normal adult MRI. However, segmentation may also be clinically necessary for MRI in cases of neurological pathology as well as for CT data. Image segmentation for application to CT images remains exceedingly important, given that much of the developing world does not have access to MRI. Additionally, much emergency imaging and many critical vascular studies in industrialized countries are done with CT. In this study, we sought

ABBREVIATIONS IRB = institutional review board; $\mathrm{NIH}=$ National Institutes of Health; $\mathrm{RF}=$ radiofrequency.

SUBMITTED September 4, 2012. ACCEPTED September 25, 2014.

INCLUDE WHEN CITING Published online November 28, 2014; DOI: 10.3171/2014.9.PEDS12426.

DISCLOSURE This work was supported by a Medical Student Fellowship grant from the American Association of Neurological Surgeons (J.G.M.), a Grace Woodward Grant for Collaborative Research in Engineering and Medicine from Penn State (S.J.S., A.G.W.), and the generosity of the endowment funds of Harvey F. Brush (S.J.S.). 
to lay the foundation for an intelligent automated edge tracker that is modality independent and has the capability to segment normative data from MRI that can be applied to both MRI and CT.

A probabilistic approach using methods developed for dynamic state tracking is an ideal technique to automatically segment noisy medical images. In engineering, the state describes the status of the system at a given instant in time. ${ }^{27}$ In this case, the system is referring to the brain edge, and variables describing that state are image intensity and local gradient in the image. A target-tracking algorithm attempts to follow changes in the system by using a model to estimate changes in state, followed by measurements of the state. It is expected that the model will not be complete enough to accurately predict changes in the state. Likewise, it is expected that there will be errors in measurement. However, when the model estimations and new measurements are combined, the resulting improved estimate is defined as the posterior estimate of state.

Fusing data with models of the state of a system-for instance, the boundary between brain and CSF-can be an optimal way to take into account noise in the data, imprecision in measurement, and inadequacy in the model and adapt all of this throughout an image. Kalman defined a filter for linear systems with Gaussian uncertainty. For systems that are not linear, and errors that are not Gaussian, a variety of particle filters have been designed. A general review can be found in Schiff, 2012.25 Target-tracking algorithms, such as the Kalman filter and the particle filter, have been successfully implemented to track the state of weather systems and control autonomous robots, systems that need to use incomplete and noisy measurements for prediction and control. ${ }^{27,29}$ These methods have the capacity to take into account any type of local and global information, making them versatile for use with many different types of data.

Research on using automatic target tracking for image segmentation has been growing. The particle filter has been used to segment cerebral arterial segments from CT angiography images by modeling an elliptical cross section of a blood vessel. ${ }^{26}$ Kalman filters have been used to extract cavity boundaries from ultrasound images, where the boundary is tracked as the distance from the center of the cavity. ${ }^{1}$ More recently, Withey et al. used a similar approach to track the edges of brain and ventricles in MR images. ${ }^{33}$ Here, the user places a seed point on the boundary of the specified tissue, and a Kalman filter is used to follow the edge until it reaches the seed point to form a closed shape.

We seek to generalize the Withey et al. algorithm with a more general approach. Unlike Kalman filters, nonparametric filters such as the particle filter do not rely on Gaussian representations of the posterior state. The posterior state here is the edge estimation after a measurement is incorporated. Rather, the particle filter approximates the posterior by a finite number of values, each a representation of the state. This makes it ideal for use in noisy medical images where noise cannot be well represented using a Gaussian probability density. The particle filter is optimized for fusing any model of the edge to be traced, making it very versatile.
Intra-brain fluid, essentially intraventricular fluid, is of particular interest to our work on obstructive hydrocephalus. Using the National Institutes of Health (NIH) pediatric MRI database, we applied the particle filter to produce normative growth curves of brain and intra-brain fluid from children and adolescents from 0 to 18 years of age. Previous studies of normative development using MRI offered an opportunity to compare our measurements to established results. Hüppi et al. measured gray matter, white matter, and CSF in normal preterm and full-term infants with gestational ages of 29-41 weeks at birth..$^{15}$ Giedd et al. measured white matter and gray matter in individuals with an age range of 4-21 years. ${ }^{13}$ Courchesne et al. measured gray matter, white matter, and CSF volumes from normal subjects with ages ranging from 19 months to 80 years. ${ }^{8}$ In these MRI studies, there exists a gap in the ages studied between birth and 19 months of age. The very rapid and substantial growth of the brain during this age range below 2 years makes this period a critical one for contrasting normative brain growth dynamics within the context of developmental dynamical diseases of the brain such as childhood hydrocephalus. ${ }^{18}$

In this first of 3 companion papers, ${ }^{16,17}$ we present a versatile tool created with the goal of segmenting brain and fluid in MR and CT images of the developing brain. We propose a novel algorithm using a particle filter to follow the boundary of the brain to segment brain and intra-brain fluid. We then describe the application of this method to produce normative growth curves for brain and fluid from MR images from children and adolescents ages 0 to 18 years and validate the segmentation of CT images of pediatric hydrocephalus. In the subsequent papers in this series, we report the application of this segmentation method and the normative growth curves to measurement of neurocognitive development in patients with hydrocephalus ${ }^{17}$ and to selection for surgery of patients with epilepsy. ${ }^{16}$

\section{Methods \\ MRI Subjects}

T2-weighted brain MRI data sets were acquired from the NIH Pediatric MRI Data Repository provided by the Montreal Neurological Institute., ${ }^{2,31}$ These images were studied under Penn State institutional review board (IRB) exemption approval, following NIH guidelines for the use of the repository images. Images had a slice thickness of either 2 or $3 \mathrm{~mm}$ depending on the protocol used, with an in-plane resolution of $1 \times 1 \mathrm{~mm}$. Normative growth curves of brain and intra-brain fluid volumes were calculated using 42 data sets, from subjects of ages 12 days to 18 years. Six data sets were analyzed at each of 7 time points: ages 2 weeks, 6 months, and 1,2,3, 6, and 18 years. A total of 34 subjects were analyzed; 6 of the subjects had scans at 2 time points and 1 subject had scans at 3 time points. Age was calculated from the expected date of birth. We then compared these growth curves to historical MRI data. ${ }^{8,13,15}$

\section{CT Subjects}

All CT DICOM images were previously acquired from the CURE Children's Hospital of Uganda. The IRB oversight for data analysis was provided by CURE, Harvard 
University, and Penn State University (with Penn State providing an exemption determination for de-identified images). One data set, with slice thickness of $2 \mathrm{~mm}$, was used to adapt particle filter parameters. Imaging data obtained in the 5 patients with hydrocephalus, slice thickness $5-8 \mathrm{~mm}$, were used to validate the segmentation.

\section{Image Segmentation}

The particle filter image segmentation algorithm consists of 3 major steps. First, a classification step creates likelihood maps of brain and fluid from the raw image data. These likelihood maps also include tissues outside the brain that are of similar intensity, such as muscle and fat (Fig. 1A). Therefore, a second step is necessary, in which a particle filter is used to track the edges of the brain map to extract brain and intra-brain fluid. Third, the initial classification step is used to segment the extracted brain and fluid, giving measurements of brain volume and intrabrain fluid volume. The technical details are represented in the Appendix, and a full working implementation of the code is available for download as a Code Archive here.

\section{Results}

\section{Simulations}

Simulated MRI data sets were used to train and evalu- ate the particle filter segmentation algorithm. Parameters for the algorithm were optimized under ideal conditions and under conditions of substantial noise and image inhomogeneity. Brain and intra-brain CSF were segmented in data sets with different levels of noise (0\%-9\%) and spatial (radiofrequency [RF]) inhomogeneity (0\%-40\%) and the Dice overlap coefficient was calculated for each region compared with ground truth. The Dice coefficient is a measure that quantifies the overlap between the segmented and ground truth volumes, and it ranges from 0 for no overlap to 1 for perfect overlap (see Appendix). Segmentation of the data typically takes approximately $2-20$ seconds per slice (on a typical desktop or laptop computer), depending on the level of noise and number of seed points needed.

\section{Parameter Optimization for MRI}

Segmentation optimization was performed with 2 data sets, one with no noise or RF inhomogeneity and another with 5\% noise and 40\% RF inhomogeneity. The calculation of the classification thresholds, the covariance in the image data, and the minimum expected tissue value were all optimized. Changing the algorithm's parameters did not change the Dice overlap coefficient substantially; however, it did affect the ease of use of the particle filter and the amount of manual intervention that was necessary.

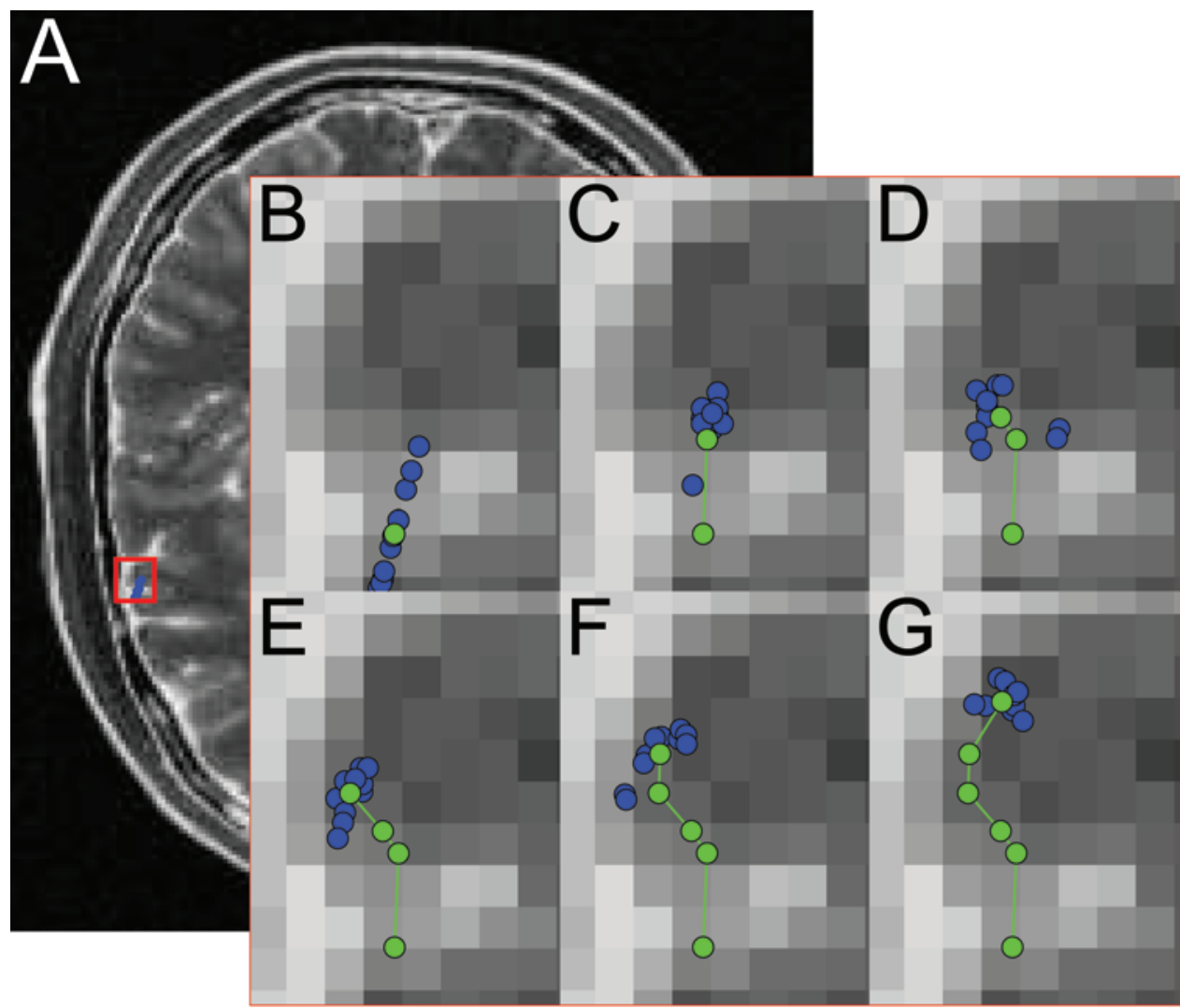

FIG. 1. The particle cloud is shown tracing a section of the brain. A: A slice of the simulated MRI with $3 \%$ noise and zero intensity RF inhomogeneity. The section being traced is outlined in red and shown enlarged in B-G. B: The particle cloud, blue, is initialized in a line surrounding the green seed point. C: The particles begin to move in the direction perpendicular to the intensity gradient and are resampled based on the likelihood of being close to the brain boundary. The brain boundary is calculated as the mean position of the particle cloud. D-G: The particles repeat this process recursively to continue tracing the brain boundary. 


\section{Segmentation Evaluation}

After the parameters were optimized, the particle filter was used to segment the simulated MR images. The particle filter is shown tracing a small section of the simulated brain in Fig. 1. The Dice overlap coefficient values and segmented volumes for each simulated data set are shown in Table 1. The Dice measure for brain ranged tightly between 0.940 and 0.946 . The Dice measure for fluid was lower, between 0.567 and 0.668 . The volumes of the ground truth brain and intra-brain fluid are 1592.67 $\mathrm{cm}^{3}$ and $85.75 \mathrm{~cm}^{3}$, respectively. The percent error ranged from $0.06 \%$ to $5.38 \%$ for brain segmentation and $2.45 \%$ to $22.34 \%$ for fluid segmentation in the simulated brain.

Although there exists a perfect ground truth for total CSF in the image, there is no proper ground truth for intra-brain CSF. The ground truth for CSF was created as any CSF within the ground truth brain, not connected to fluid outside the brain. Even without noise and RF inhomogeneity added to the simulation, the MRI simulation itself was enough to connect and disconnect boundaries between intra- and extra-brain fluid in many of the slices, making it impossible for the segmented intra-brain fluid to match perfectly with the ground truth.

\section{Parameter Optimization for CT}

Parameter optimization was performed with a manually segmented CT data set to generate a ground truth image (see Appendix for construction details). The parameters were optimized by comparing the particle filter segmented data set to the ground truth manually segmented images using the Dice overlap coefficient.

\section{Normative Growth Curves}

Normative growth curves of brain and intra-brain volumes were created from 42 MRI data sets, obtained at ages 2 weeks, 6 months, and 1,2, 3, 6, and 18 years. The volumes of brain and fluid as a function of age are shown in Fig. 2A and C, separated into male and female curves. Normative head circumference is also shown for comparison in Fig. 2B. The brain volume grows rapidly up to 2 years of age, after which the brain grows more slowly. The male brain grows faster than the female brain, a function of body size ${ }^{14}$ and is larger at age 18 . The fluid volume increases until 2 years of age, then remains approximately the same. Sex has a smaller effect in the differences of

TABLE 1. Dice coefficient and segmented volumes for each simulated data set

\begin{tabular}{|c|c|c|c|c|c|}
\hline \multirow{2}{*}{$\begin{array}{c}\% \\
\text { Noise }\end{array}$} & \multirow[b]{2}{*}{$\%$ RF } & \multicolumn{2}{|c|}{ Dice Coefficient } & \multicolumn{2}{|c|}{ Volume $\left(\mathrm{cm}^{3}\right)$} \\
\hline & & Brain & Fluid & Brain & Fluid \\
\hline \multirow[t]{2}{*}{0} & 20 & 0.946 & 0.597 & 1591.56 & 66.81 \\
\hline & 40 & 0.943 & 0.668 & 1666.29 & 83.65 \\
\hline \multirow[t]{2}{*}{1} & 0 & 0.944 & 0.612 & 1634.14 & 77.20 \\
\hline & 20 & 0.946 & 0.632 & 1630.04 & 72.67 \\
\hline \multirow[t]{2}{*}{3} & 0 & 0.940 & 0.648 & 1678.42 & 69.80 \\
\hline & 40 & 0.943 & 0.664 & 1665.23 & 81.56 \\
\hline 7 & 0 & 0.940 & 0.567 & 1583.75 & 89.52 \\
\hline 9 & 0 & 0.941 & 0.600 & 1584.20 & 66.59 \\
\hline
\end{tabular}

fluid volumes. The normative head circumference curves show a similar shape to the brain volume curves. However, while brain volume growth levels off after 2 years of age, head circumference continues to increase almost linearly up to 18 years; indeed, the typical $1-\mathrm{cm}$ thickening of the skull, scalp, and muscle during later childhood likely adds an additional several centimeters to head circumference not reflective of brain growth. The coefficient of multiple determination, $\mathrm{R}^{2}$, was used to test the effectiveness of a fit to a power law curve of the form $y=A x^{b}$, where $0<b<$ 1. $\mathrm{R}^{2}$ is a measure of goodness-of-fit and is the ratio of the regression sum of squares to the total sum of squares. ${ }^{23}$ It quantifies how much of the variance in volume is explained by the given model. The $\mathrm{R}^{2}$ values of the least-squares fitted curves (solid lines in Fig. 2A) for normative male and female brain volumes are 0.86 and 0.90 , respectively, reflecting a very good fit. For normative male and female fluid volumes, the values are both 0.25 . Although our simple power-law growth model fit the brain growth curves well, it did not account well for CSF; indeed, CSF does not grow per se during development, and would not be expected to follow a biologically based model of growth.

Additionally, we compared our brain and fluid volumes to historical MRI data from Courchesne et al., ${ }^{8}$ Giedd et al., ${ }^{13}$ and Hüppi et al. ${ }^{15}$ Our calculated brain growth curves for males (blue) and females (red), mean (solid lines) and variance (shaded regions), are superimposed over brain volume data from these 3 prior papers (markers) in Fig. 3 , showing excellent consistency and bridging the gap of development between full-term infants shown in Hüppi et al..$^{15}$ and 2-year-olds shown in Courchesne et al. ${ }^{8}$ It is more difficult to compare our fluid volumes with previous work, as we are measuring intra-brain CSF, while Hüppi et al. ${ }^{15}$ and Courchesne et al. ${ }^{8}$ measured total CSF. Giedd et al..$^{13}$ did not report CSF volumes. We obtained intra-brain CSF volumes that are less than the total CSF volumes reported in the previous 2 papers, as expected since we focused on intra-brain fluid. Intra-brain CSF will dilate the brain in hydrocephalus and have a direct effect on neurocognitive outcome (as shown in the second paper in this series ${ }^{17}$ ), and in the evaluation of structural brain pathology such as temporal lobe epilepsy, parenchymal volume loss is related to intraventricular volume gain, as discussed in the third paper in this series. ${ }^{16}$ Were there a need to extend this method to communicating hydrocephalus, additional steps would be needed to obtain external fluid volumes.

\section{Segmentation of Pediatric Hydrocephalus CT Images}

We segmented 5 CT data sets of pediatric hydrocephalus. We employed the parameters optimized using our constructed CT ground truth image (see Appendix). A sample segmentation is shown in Fig. 4 (Fig. 4A is the CT image, Fig $4 \mathrm{~B}$ is the segmented brain tissue, and Fig. $4 \mathrm{C}$ is the segmented intrabrain fluid). The adaptations needed to use the particle filter algorithm with CT data were minimal and consisted only of parameter adjustments. The correspondence of brain and fluid volume as calculated by the particle filter and manual segmentation using the Cavalieri technique is shown in Fig. 5. The correlation coefficient (the Pearson product-moment correlation coefficient, calculated as the covariance of the 2 variables 

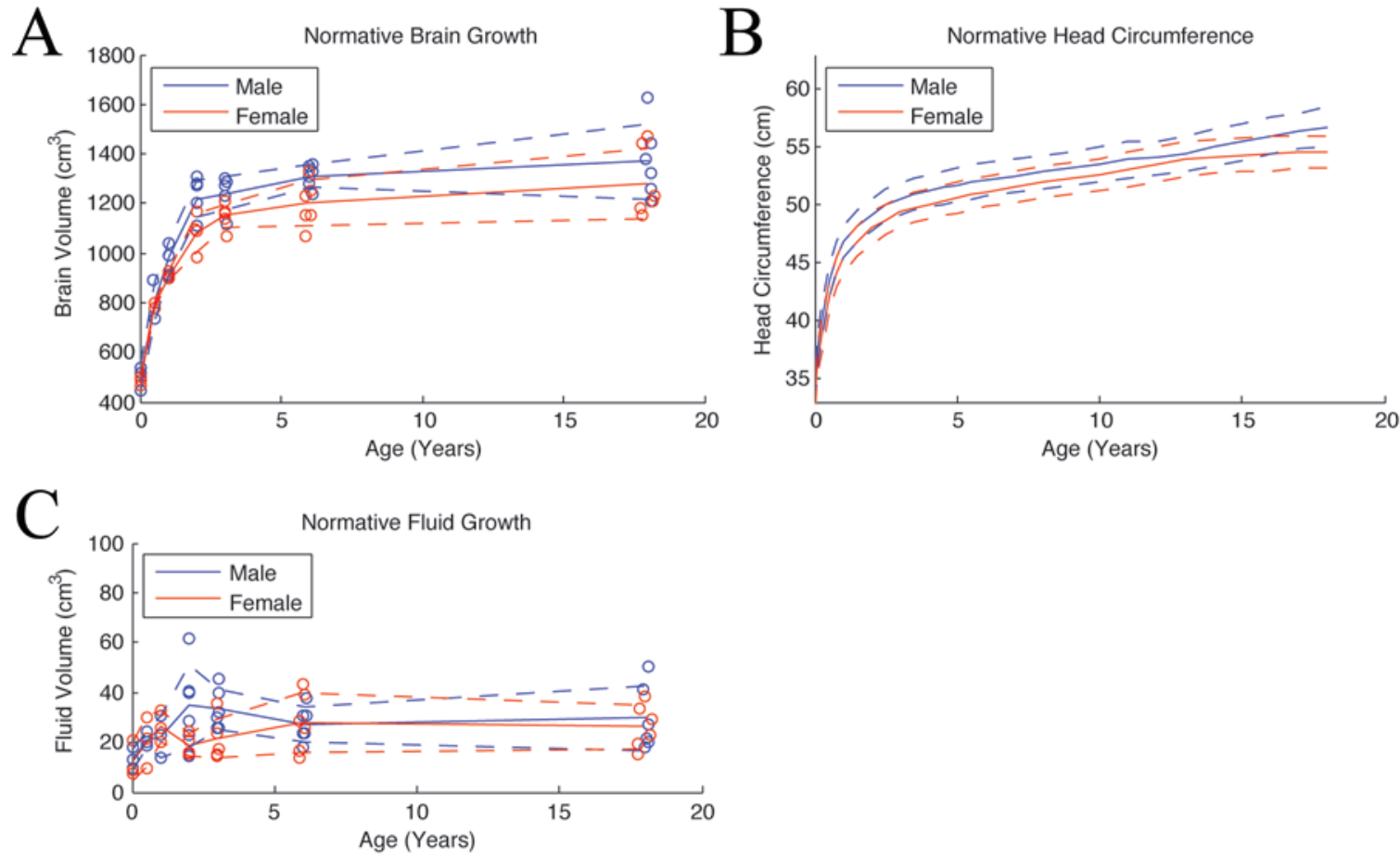

FIG. 2. Normative growth curves for male (blue) and female (red). The solid lines are the mean measurements, while the dotted lines represent \pm 1 SD. The open circles show individual data points. Normative brain volume $(\mathrm{A})$ and fluid volume $(\mathrm{C})$ from the $\mathrm{NIH}$ Pediatric MRI Data Repository. Normative head circumference data (B) from Roche et al. ${ }^{24}$ are shown for comparison.

divided by the product of the variances), $r$, was 0.993 for brain measurements $(\mathrm{p}<0.001)$ and 0.997 for fluid measurements $(\mathrm{p}<0.001)$, demonstrating excellent correspondence between volumes calculated using the particle filter and the manual Cavalieri method.

\section{Discussion}

In this study, we employ a robust target-tracking algorithm, the particle filter, as a versatile automated imagesegmentation tool. The algorithm was validated with a simulated data set of T2-weighted MR images of the brain.

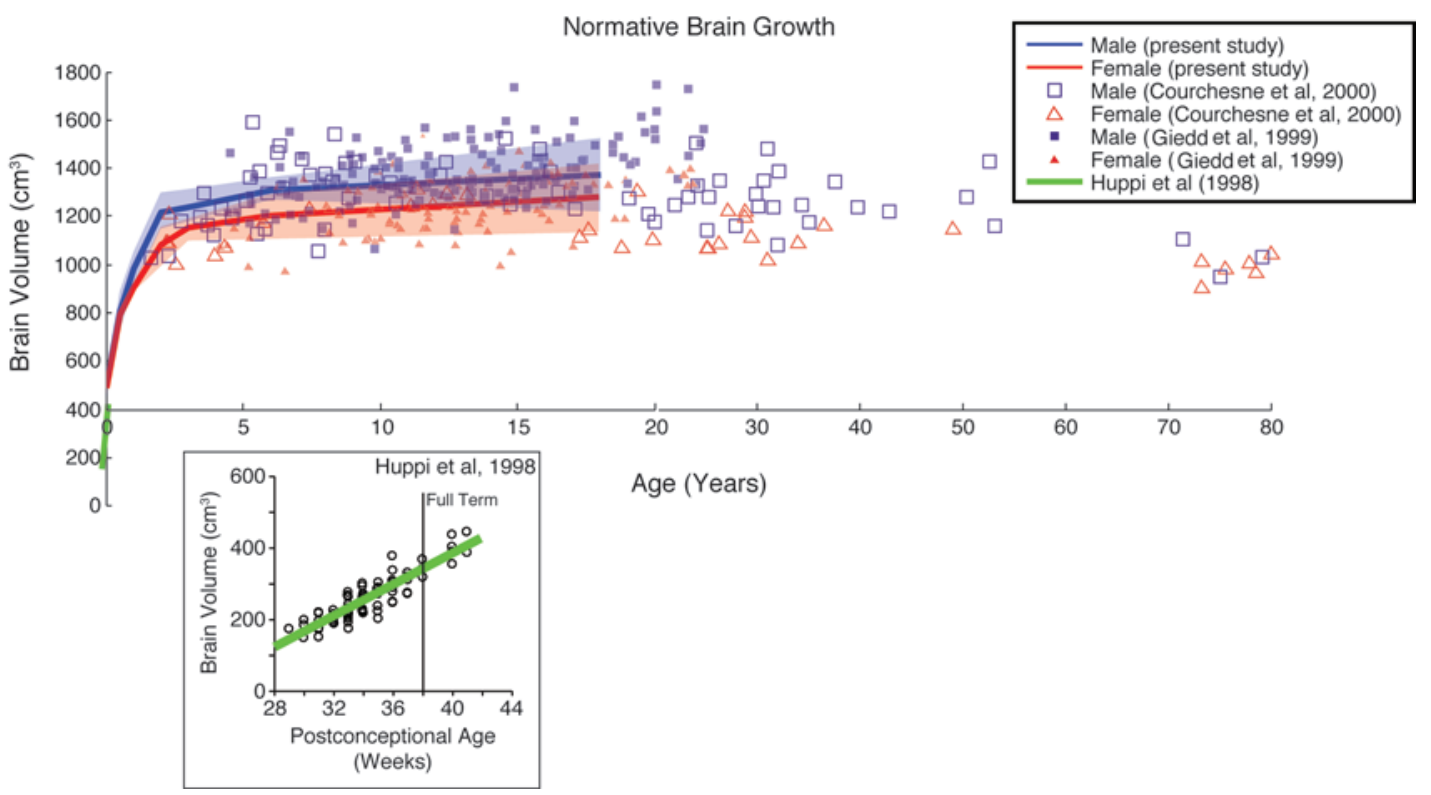

FIG. 3. Normative brain growth curves calculated in this study are shown overlain with historical MRI data. Our data are shown as solid lines with the shaded region displaying $\pm 1 \mathrm{SD}$ from the mean. Open shapes represent volumes from Courchesne et al. ${ }^{8}$ Closed shapes represent volumes from Giedd et al..$^{13}$ The solid green line represents the mean volume curve from Hüppi et al. ${ }^{15}$ of 100 preterm and full-term infants with gestational age at birth of 29-41 weeks. The insert shows the data from Hüppi et al. on enlarged axes, with the mean line in green and individual data points as open black circles. 

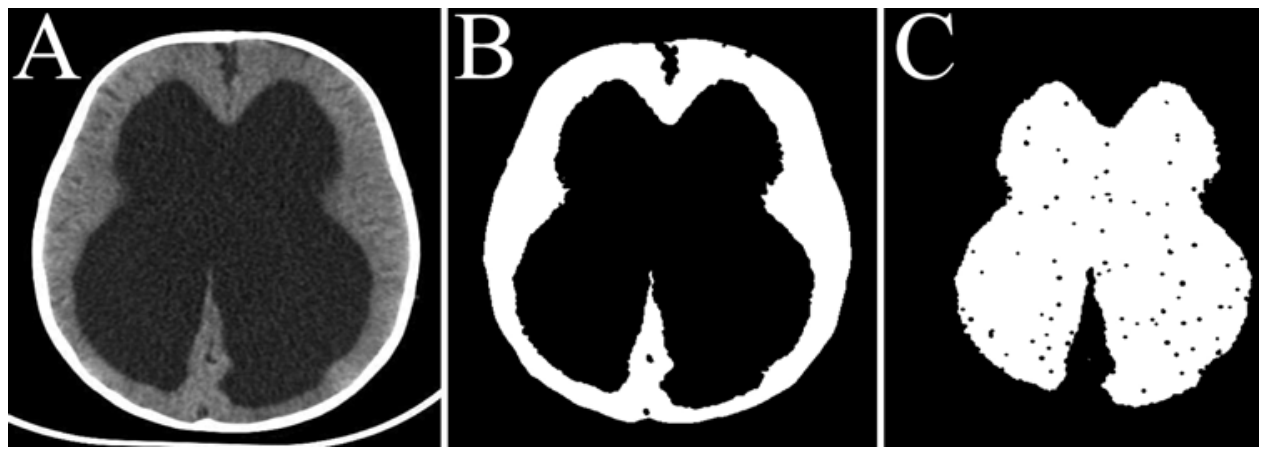

FIG. 4. Axial CT image obtained in a 6-month-old infant with severe hydrocephalus. Panel A is the original CT slice, Panel B is the segmented brain, and Panel $\mathbf{C}$ is the segmented fluid. Image and data courtesy of $B$. Warf.

The particle filter segmentation tool was then used to create normative growth curves of brain and fluid volumes using the NIH Pediatric MRI Data Repository. We validated the particle filter with CT images by measuring brain and fluid volumes in 5 data sets of pediatric hydrocephalus with the particle filter and manual Cavalieri methods.

\section{Optimization}

Parameters for the particle filter were optimized by segmenting 2 simulated ground truth data sets, one without noise or RF inhomogeneity and the other with $5 \%$ noise and $40 \%$ RF inhomogeneity.

Our method does require user interference in the segmentation. We did not completely analyze the effects of different initial inputs on the results of the final segmentation. Nevertheless, the effects of user input did seem to be low. For example, if the user failed to place the seed point close enough to the boundary, the particle cloud did not move forward and the user simply selected another seed point. Additionally, variability of results between users was not fully explored; it will be important to study intraand interuser reliability in future work.

\section{Evaluation}

The particle filter segmentation tool was evaluated on simulated MRI data sets ranging in noise from $0 \%$ to $9 \%$ and RF inhomogeneity from $0 \%$ to $40 \%$. The algorithm was reasonably robust to large amounts of random noise and RF inhomogeneities. Images with large RF inhomogeneities could be preprocessed to remove these intensity gradients. ${ }^{4}$ It is important to note, however, that a $40 \%$ $\mathrm{RF}$ inhomogeneity for brain scans at low field (1.5-3.0 T) is very large. Field strength inhomogeneities of $40 \%$ for brain scans are more commonly seen at $7 \mathrm{~T} .{ }^{30}$ The data used in this study from the NIH pediatric MRI database were all acquired at $1.5 \mathrm{~T}$, where an RF inhomogeneity of $5 \%$ is expected. We recognize that while segmenting brain images with $40 \%$ inhomogeneities will be uncommon, especially in the clinical setting, it is nevertheless important to probe the performance of the segmentation tool under more extreme circumstances.

The particle filter segmented brain tissue more accurately than fluid. This, however, is expected given that there is much more brain to segment than fluid, which results in a smaller percent error for each misclassified voxel. Additionally, the ground truth image for CSF that was used was not a perfect representation of intra-brain CSF and was calculated not by anatomy, but by pixel connectivity prior to the MRI simulation. Although the evaluation process was not as accurate for fluid segmentation, the results were
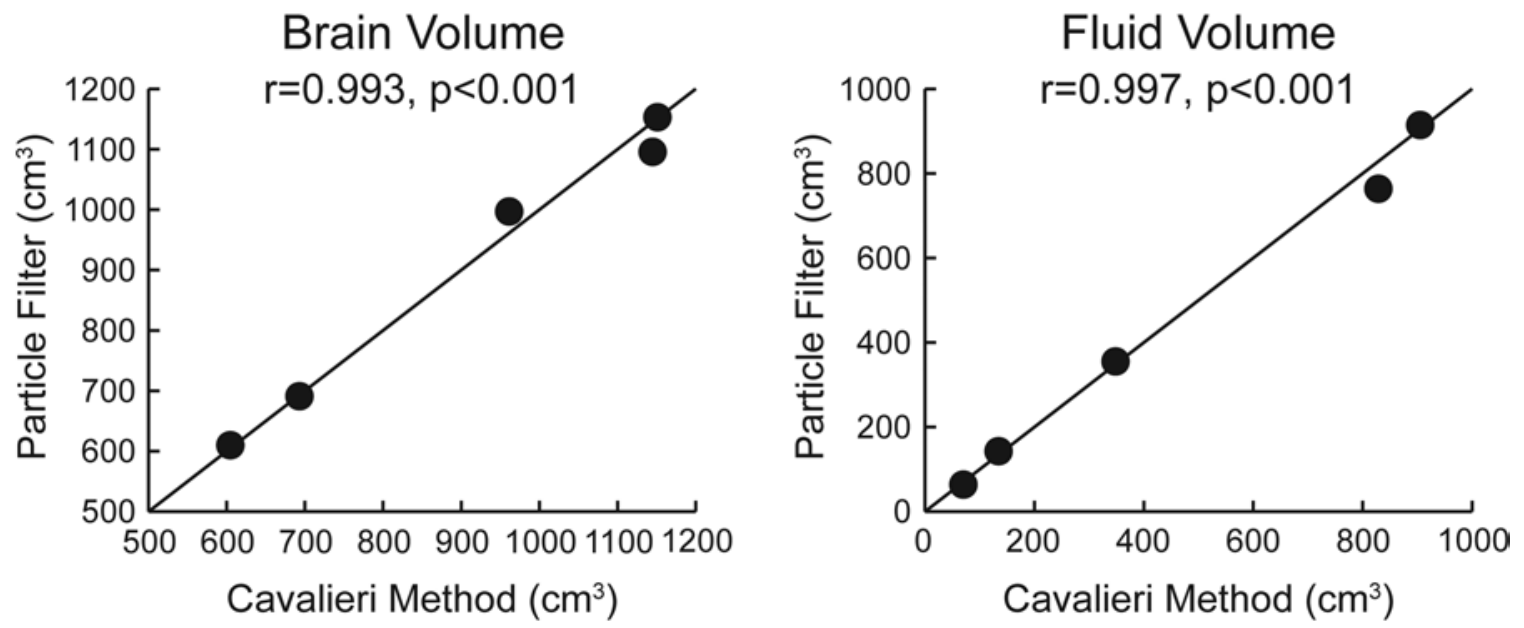

FIG. 5. Brain (left) and fluid (right) volumes are plotted as measured by the particle filter and by the manual Cavalieri method. Equal volume measurements would be plotted on the solid diagonal line, shown for reference. 
visually inspected by a neurosurgeon (S.J.S.) to ensure acceptable results (Videos 1 and 2).

VIDEO 1. Video demonstrating the particle filter tracking the edge of the brain in a CT scan obtained in a 12-year-old child. This particular image slice had 3 disconnected regions of brain and required 3 initiation points to completely segment the brain. Copyright Steven J. Schiff and Jason G. Mandell. Published with permission. Click here to view with Media Player. Click here to view with Quicktime.

VIDEO 2. Video demonstrating the particle filter tracking the edge of the brain in a T2-weighted MR image obtained in a 6-year-old child. The brain was fully connected on this slice, and only 1 initiation point was required for the segmentation. Copyright Steven J. Schiff and Jason G. Mandell. Published with permission. Click here to view with Media Player. Click here to view with Quicktime.

\section{Growth Curves}

Normative growth curves of brain and fluid volume were created from the NIH Pediatric MRI Database (Fig. 2). Normative head circumference curves for males and females are shown in Fig. 2 for comparison. ${ }^{24}$ As expected, brain volume development shows similarities in shape to head circumference development for the normal child. Males measure slightly larger than females in both curves as a function of body size ${ }^{14}$ and there is rapid growth up to 2 years of age. However, whereas brain volume growth slows after 2 years of age, head circumference increases linearly up to 18 years of age. It is likely that some of the increase in head circumference throughout childhood is due to thickening of skull, muscle, and scalp, rather than brain. There were very small differences seen in fluid volumes between males and females. Reiss et al. ${ }^{22}$ have also shown this with a larger population, although this prior study was of children aged 5-17 years, and no evidence of brain growth with age was discernable in the findings.

We also show our brain growth curves overlain with data from Courchesne et al., ${ }^{8}$ Giedd et al. ${ }^{13}$ and Hüppi et al. ${ }^{15}$ in Fig. 3 to show additional validation of our brain volume measurements. Each of these studies used MRI to calculate brain volumes at different ages. In this study, we show brain volumes over the very rapidly developing age range of birth to 2 years of age, which is not present in these previous studies, and connect this to the remainder of brain development through 18 years. Normative volume development at these ages is essential for studying brain volume development in neurological disorders of pediatric patients.

\section{Pediatric Hydrocephalus Segmentation}

Warf et al. ${ }^{32}$ acquired CT scans and administered the Bayley Scales of Infant and Toddler Development, third edition (BSID-III) to African pediatric patients treated for hydrocephalus. We segmented 5 data sets from this study to calculate brain and fluid volumes. We show a very significant linear correlation between brain and fluid volumes as measured by the particle filter and with the manually segmented Cavalieri method. Additionally, very little bias is seen using the particle filter. If we are to understand and treat hydrocephalus and other neurological disorders with volumetry in the developing world, we need to be able to segment CT data quickly and reliably.

\section{Conclusions}

This study represents the first use of a particle filter as an edge tracker in image segmentation, and offers a semiautomatic method to segment both pediatric and adult brain data from MR and CT images. It is quite feasible for clinicians to use this method to calculate brain and fluid volumes as diagnostic measures; nevertheless, extending our efforts to a true 3D segmentation tool, with only one seed point required, would improve usability and potentially increase accuracy. ${ }^{33}$ Additionally, this method is very expandable. We use a very simple model of the brain boundary. The particle filter could easily be combined with a probabilistic atlas of the whole brain or for specific brain structures. Furthermore, the particle filter could be combined with different pixel classification methods and results from region growing techniques. ${ }^{33}$

We applied the particle filter to create normative growth curves for brain and fluid volumes to study development between birth and 18 years of age. The brain volumes measured are consistent with previous work and add to the literature by more completely quantifying development between birth and 18 years of age. Study of this extended age range is necessary to understand differences in early brain and fluid growth in pediatric neurological diseases. The particle filter offers the tools to quickly and easily measure brain and fluid volumes over a wide variety of data, and indeed, studies using the particle filter to understand postsurgical outcome in pediatric hydrocephalus and epilepsy are reported in the companion papers that follow as parts 2 and $3 .^{16,17}$

\section{Acknowledgments}

We are deeply grateful to B. Warf, A. Kulkarni, and B. K. Nsubuga for sharing the hydrocephalus image examples with us.

\section{References}

1. Abolmaesumi P, Sirouspour MR: An interacting multiple model probabilistic data association filter for cavity boundary extraction from ultrasound images. IEEE Trans Med Imaging 23:772-784, 2004

2. Almli CR, Rivkin MJ, McKinstry RC: The NIH MRI study of normal brain development (Objective-2): newborns, infants, toddlers, and preschoolers. Neuroimage 35:308-325, 2007

3. Bankman IN: Handbook of Medical Image Processing and Analysis, ed 2. Burlington, MA: Academic Press, 2009

4. Bazin PL, Pham DL: Topology-preserving tissue classification of magnetic resonance brain images. IEEE Trans Med Imaging 26:487-496, 2007

5. Briellmann RS, Pell GS, Wellard RM, Mitchell LA, Abbott DF, Jackson GD: MR imaging of epilepsy: state of the art at $1.5 \mathrm{~T}$ and potential of $3 \mathrm{~T}$. Epileptic Disord 5:3-20, 2003

6. Cocosco CA, Kollokian V, Kwan RKS, Evans AC: BrainWeb: online interface to a 3D MRI simulated brain database. Proceedings of 3rd International Conference on Functional Mapping of the Human Brain, Copenhagen, May 1997. NeuroImage 5:S425, 1997 (Abstract)

7. Collins DL, Zijdenbos AP, Kollokian V, Sled JG, Kabani NJ, Holmes CJ, et al: Design and construction of a realistic digital brain phantom. IEEE Trans Med Imaging 17:463-468, 1998

8. Courchesne E, Chisum HJ, Townsend J, Cowles A, Covington J, Egaas B, et al: Normal brain development and aging: quantitative analysis at in vivo MR imaging in healthy volunteers. Radiology 216:672-682, 2000

9. Deans SR: The Radon Transform and Some of Its Applications. New York: John Wiley \& Sons, 1983 
10. Dice LR: Measures of the amount of ecologic association between species. Ecology 26:297-302, 1945

11. Drew PJ, Blinder P, Cauwenberghs G, Shih AY, Kleinfeld D: Rapid determination of particle velocity from space-time images using the Radon transform. J Comput Neurosci 29:5-11, 2010

12. Flury B: A First Course in Multivariate Statistics. New York: Springer, 1997

13. Giedd JN, Blumenthal J, Jeffries NO, Castellanos FX, Liu $\mathrm{H}$, Zijdenbos A, et al: Brain development during childhood and adolescence: a longitudinal MRI study. Nat Neurosci 2:861-863, 1999

14. Gould SJ: The Mismeasure of Man. New York: Norton, 1981

15. Hüppi PS, Warfield S, Kikinis R, Barnes PD, Zientara GP, Jolesz FA, et al: Quantitative magnetic resonance imaging of brain development in premature and mature newborns. Ann Neurol 43:224-235, 1998

16. Mandell JG, Hill KL, Nguyen DTD, Moser KW, Harbaugh RE, McInerney J, et al: Volumetric brain analysis in neurosurgery: Part 3. Volumetric CT analysis as a predictor of seizure outcome following temporal lobectomy. J Neurosurg Pediatr [epub ahead of print November 28, 2014. DOI: 10.3171/2014.9.PEDS12428]

17. Mandell JG, Kulkarni AV, Warf BC, Schiff SJ: Volumetric brain analysis in neurosurgery: Part 2. Brain and CSF volumes discriminate neurocognitive outcomes in hydrocephalus. J Neurosurg Pediatr [epub ahead of print November 28, 2014. DOI: 10.3171/2014.9.PEDS12427]

18. Mandell JG, Neuberger T, Drapaca CS, Webb AG, Schiff SJ: The dynamics of brain and cerebrospinal fluid growth in normal versus hydrocephalic mice. Laboratory investigation. J Neurosurg Pediatr 6:1-10, 2010

19. Matsumae M, Kikinis R, Mórocz I, Lorenzo AV, Albert MS, Black PM, et al: Intracranial compartment volumes in patients with enlarged ventricles assessed by magnetic resonance-based image processing. J Neurosurg 84:972-981, 1996

20. Mortensen EN, Barrett WA: Interactive segmentation with intelligent scissors. Graph Models Image Proc 60:349-384, 1998

21. Pham DL, Xu C, Prince JL: Current methods in medical image segmentation. Annu Rev Biomed Eng 2:315-337, 2000

22. Reiss AL, Abrams MT, Singer HS, Ross JL, Denckla MB: Brain development, gender and IQ in children. A volumetric imaging study. Brain 119:1763-1774, 1996

23. Rencher AC: Methods of Multivariate Analysis, ed 2. New York: John Wiley \& Sons, 2002

24. Roche AF, Mukherjee D, Guo SM, Moore WM: Head circumference reference data: birth to 18 years. Pediatrics 79:706-712, 1987

25. Schiff SJ: Neural Control Engineering: The Emerging Intersection between Control Theory and Neuroscience. Cambridge, MA: Massachusetts Institute of Technology Press, 2012

26. Shim H, Kwon D, Yun ID, Lee SU: Robust segmentation of cerebral arterial segments by a sequential Monte Carlo method: particle filtering. Comput Methods Programs Biomed 84:135-145, 2006

27. Simon D: Optimal State Estimation. Hoboken, NJ: John Wiley \& Sons, Inc, 2006

28. Sonka M, Hlavac V, Boyle R: Image Processing, Analysis, and Machine Vision, ed 3. Stamford, CT: Cengage Learning, 2007

29. Thrun S, Burgard W, Fox D: Probabilistic Robotics. Cambridge, MA: Massachusetts Institute of Technology Press, 2006

30. Vaughan JT, Garwood M, Collins CM, Liu W, DelaBarre L, Adriany G, et al: 7T vs. 4T: RF power, homogeneity, and signal-to-noise comparison in head images. Magn Reson Med 46:24-30, 2001

31. Waber DP, De Moor C, Forbes PW, Almli CR, Botteron
KN, Leonard G, et al: The NIH MRI study of normal brain development: performance of a population based sample of healthy children aged 6 to 18 years on a neuropsychological battery. J Int Neuropsychol Soc 13:729-746, 2007

32. Warf B, Ondoma S, Kulkarni A, Donnelly R, Ampeire M, Akona J, et al: Neurocognitive outcome and ventricular volume in children with myelomeningocele treated for hydrocephalus in Uganda. Clinical article. J Neurosurg Pediatr 4:564-570, 2009

33. Withey DJ, Pedrycz W, Koles ZJ: Dynamic edge tracing: boundary identification in medical images. Comput Vis Image Underst 113:1039-1052, 2009

34. Zou KH, Warfield SK, Bharatha A, Tempany CMC, Kaus MR, Haker SJ, et al: Statistical validation of image segmentation quality based on a spatial overlap index. Acad Radiol 11:178-189, 2004

\section{Author Contributions}

Conception and design: all authors. Acquisition of data: Schiff, Mandell, Langelaan. Analysis and interpretation of data: all authors. Drafting the article: all authors. Critically revising the article: all authors. Reviewed submitted version of manuscript: all authors. Approved the final version of the manuscript on behalf of all authors: Schiff. Statistical analysis: Schiff, Mandell, Langelaan. Administrative/technical/material support: Schiff, Langelaan, Webb. Study supervision: Schiff, Langelaan, Webb.

\section{Supplemental Information}

\section{Companion Papers}

Mandell JG, Kulkarni AV, Warf BC, Schiff SJ: Volumetric brain analysis in neurosurgery: Part 2. Brain and CSF volumes discriminate neurocognitive outcomes in hydrocephalus. DOI: 10.3171/2014.9.PEDS12427.

Mandell JG, Hill KL, Nguyen DTD, Moser KW, Harbaugh RE, McInerney J, et al: Volumetric brain analysis in neurosurgery: Part 3. Volumetric CT analysis as a predictor of seizure outcome following temporal lobectomy. DOI: 10.3171/2014.9.PEDS12428.

\section{Videos}

Video 1, Media Player. http://mfile.akamai.com/21490/wmv/ digitalwbc.download.akamai.com/21492/wm.digitalsource-naregional/peds12-426_video_1.asx.

Video 1, Quicktime. http://mfile.akamai.com/21488/mov/ digitalwbc.download.akamai.com/21492/qt.digitalsource-global/ peds12-426_video_1.mov.

Video 2, Media Player. http://mfile.akamai.com/21490/wmv/ digitalwbc.download.akamai.com/21492/wm.digitalsource-naregional/peds12-426_video_2.asx.

Video 2, Quicktime. http://mfile.akamai.com/21488/mov/ digitalwbc.download.akamai.com/21492/qt.digitalsource-global/ peds12-426_video_2.mov.

\section{Code Archive}

http://thejns.org/userimages/ContentEditor/1393517259171/ Supplementary\%20Data\%20Mandell\%20et\%20al\%20Paper\%20I. zip.

\section{Correspondence}

Steven J. Schiff, W311 Millennium Science Complex, Pollock Rd., University Park, PA 16802-2131. email: sschiff@psu.edu.

\section{Appendix Classification}

The first step in the segmentation process is a classification step, during which maps of brain likelihood and fluid likelihood are pro- 
duced from the raw MRI data based on user-selected pixels of air, brain, and fluid (Appendix Fig. 1).

Three slices are displayed to the user, corresponding to the first, second, and third quartiles of the image stack. The user is asked to select 10 voxels in these slices from each of 3 categories -1) air, 2) gray and white matter, and 3) fluid-for a total of 30 voxels. These points serve to calculate 2 intensity boundaries: $t_{1}$, the boundary between air and brain; and $t_{2}$, the boundary between brain and fluid. $t_{1}$ is calculated as the mean of the median intensity of the air voxels and the minimum brain voxel intensity. $t_{2}$ is the mean of the maximum brain intensity selected and the median fluid intensity. This process could be further automated, and the variability of the segmentation seemed to be low as long as the selected pixels were within tissue boundaries.

The intensity thresholds are used for classification by assigning likelihoods that a voxel contains a given tissue-brain or fluidbased on that voxel's neighborhood. A $5 \times 5$ mask with 13 non-zero unitary elements is created to define the pixel neighborhood (Eq. 1). For each pixel in the image, the likelihood that a pixel contains a given tissue is estimated as the fraction of pixels in the neighborhood with an intensity within the tissue region. This fraction is squared to increase contrast for the particle filter.

$$
\operatorname{mask}=\left[\begin{array}{ccccc}
0 & 0 & 1 & 0 & 0 \\
0 & 1 & 1 & 1 & 0 \\
1 & 1 & 1 & 1 & 1 \\
0 & 1 & 1 & 1 & 0 \\
0 & 0 & 1 & 0 & 0
\end{array}\right]
$$

\section{Edge Tracing}

The edge-tracing step of the algorithm extracts the brain from surrounding tissue by tracing the brain boundary. The edge tracer segments the brain likelihood map, while the original slice is shown to the user for accurate observation and intervention. During this step in the algorithm, the $x$ - and $y$-components of the 2D intensity gradient are first calculated from the brain map after a Gaussian filter is applied $(3 \times 3$ pixel neighborhood, standard deviation, $\sigma$ $=3$ ). Then, the user selects a seed point. Particles are initialized at the seed point and given a velocity in the direction of the boundary. Particles are then propagated along the boundary of the brain until a closed shape has been formed.

\section{Particle State}

To track the boundary of the brain, the particle filter represents the likelihood that a particular region of the image contains a brain boundary. Each particle (located at $x_{n}, y_{n}$ ) computes the likelihood that the brain boundary lies on that pixel. The $N$ particles are described by their state, $x_{n}$, where $n=[1,2, \ldots, N]$.

$$
x_{n}=\left[\begin{array}{llll}
\dot{x}_{n} & \dot{y}_{n} & x_{n} & y_{n}
\end{array}\right]^{T}
$$

where, $\dot{x}_{n}$ and $\dot{y}_{n}$ describe the particle's velocity in the $x$ and $y$ directions, respectively, $x_{n}$ and $y_{n}$ define the particle's position, and $T$ indicates transpose.

\section{Particle Filter Initialization}

Particles are generated with positions on a line perpendicular to the brain boundary with a Gaussian distribution centered on the seed point with a standard deviation of 1.5. The direction of the edge is calculated using a Radon transform of the gradient magnitude image in the $11 \times 11$ neighborhood of the seed point (Appendix Fig. 2). The Radon transform calculates a projection of the image intensity along a line oriented at angles from 0 to $\pi$ radians. ${ }^{9}$ The projection perpendicular to the edge will have the least uniform intensity, and thus the highest variance. ${ }^{11}$

The particle velocities are initialized in the direction of the edge with a speed of 1 pixel/time-step - slow enough to maintain detailed resolution, but fast enough for reasonable computation time.

\section{Particle Filter}

The particle filter consists of 4 major steps that are repeated until a closed shape is formed (Appendix Fig. 3):

1. Use motion model to propagate particles forward in time.

2. Take measurements at new particle locations.

3. Compute the likelihood that a particle is on the brain boundary by using the brain likelihood map and the $2 \mathrm{D}$ gradient images.

4. Resample based on this likelihood (i.e., select particles with the highest weight, discard those with low weight).

Step 1: The velocity of each particle is defined in the direction perpendicular to its local gradient with a speed proportional to the gradient strength. For each time-step, $k$, the particles are propagated forward using the process equation,

$$
\begin{gathered}
x_{k+1}=A x_{k}+w_{k} \\
A=\left[\begin{array}{llll}
1 & 0 & 0 & 0 \\
0 & 1 & 0 & 0 \\
1 & 0 & 1 & 0 \\
0 & 1 & 0 & 1
\end{array}\right]
\end{gathered}
$$

where $w_{k}$ is the 0 -mean, Gaussian process noise of standard devia-
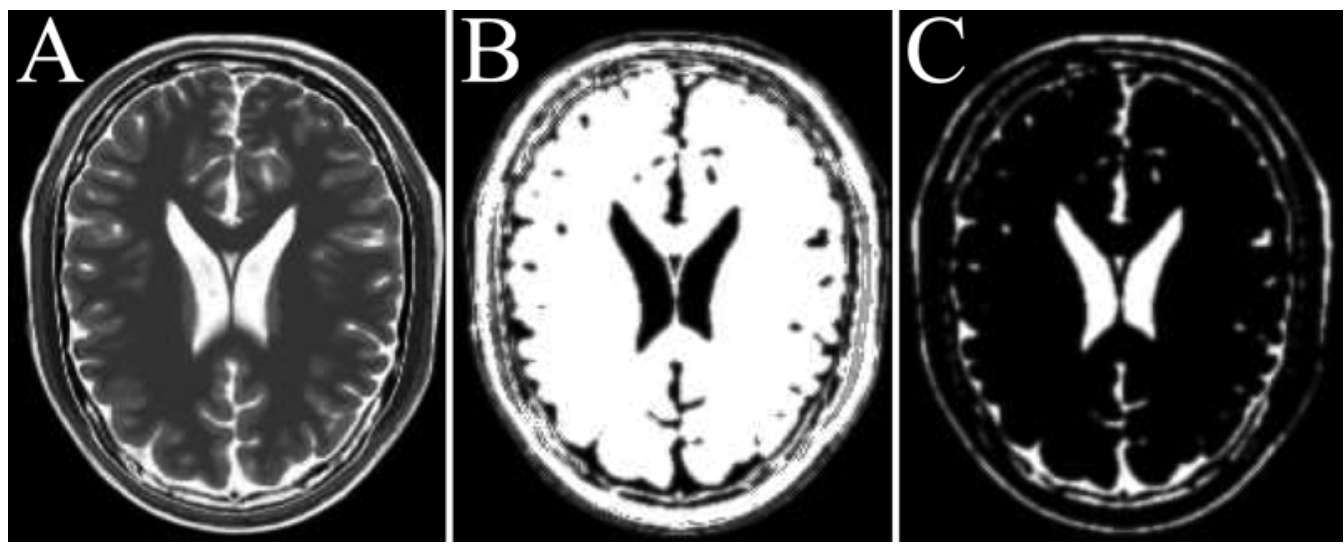

APPENDIX FIG. 1. Classification of a typical image slice. A: The original image. B: The calculated brain likelihood map. C: The calculated fluid likelihood map. 

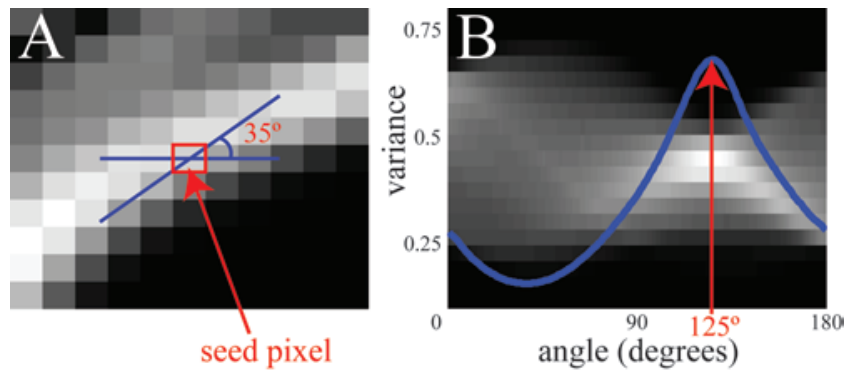

APPENDIX FIG. 2. A: The $11 \times 11$ area surrounding the seed point (outlined in red) of the gradient magnitude of the brain likelihood map. B: The Radon transform of the image on the left with the variance at each degree overlaid in blue. The degree of the projection is on the abscissa and the variance is on the ordinate. The angle with the largest variance is perpendicular to the edge.

tion $0.05 . A$ is the process matrix, defined to update the new position as the sum of the previous position and velocity multiplied by a time step of 1 .

Step 2: The particle filter predicts the next measurement before actually taking the next measurement. The prediction and the actual measurement are then compared with the question: what is the probability that the predicted measurement has occurred given the actual measurement? In this algorithm, the prediction is the ideal brain edge and does not need to change. We therefore ask, what is the probability that we have the ideal brain edge given the actual measurement. The ideal brain edge has a maximum gradient, with minimum and maximum intensities in its pixel neighborhood. The predicted measurement, $z^{*}$, is defined:

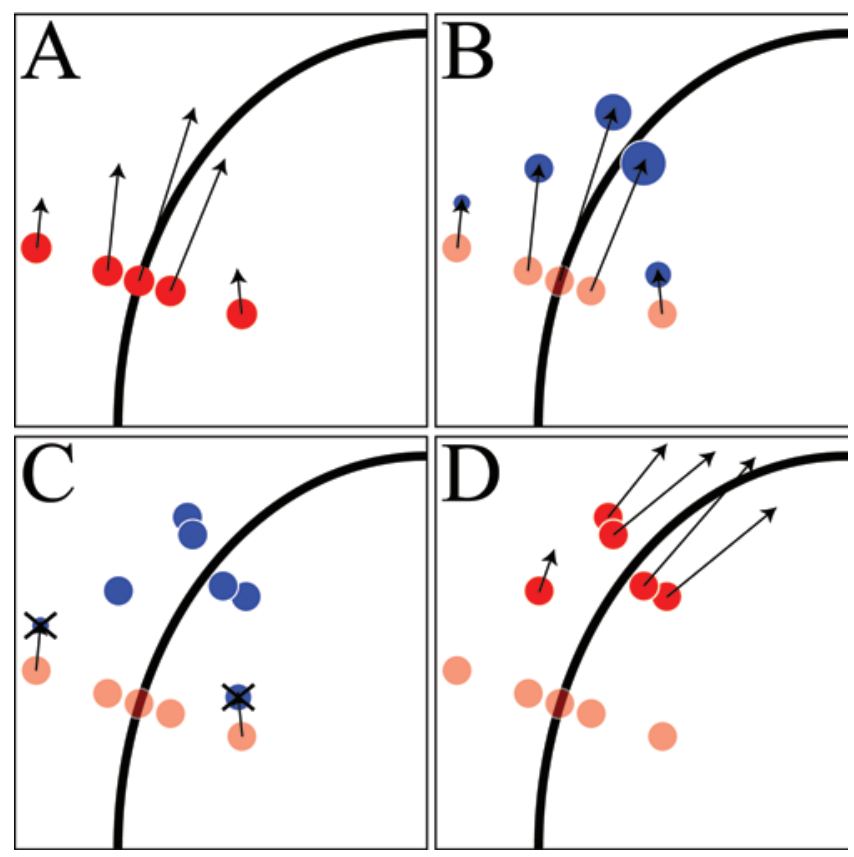

APPENDIX FIG. 3. The particle filter applied to the problem of edge tracing: A: The particles are propagated forward in time based on their local gradient. B: Measurements are taken at the new particle locations and each particle is weighted based on the likelihood that it is on the edge, shown as particle size. C: Particles are resampled based on weight, particles of highest weight are retained and possibly duplicated, and noise is added to particle position, while particles of lowest weight are dropped $\mathbf{D}$ : The process continues recursively and the new particles are propagated forward.

$$
z^{*}=\left[\nabla_{x, \text { max }} \nabla_{y, \text { max }} L_{\text {max }, \text { global }} L_{\text {min, global }}\right]^{T}
$$

where $\nabla_{x, \text { max }}$ and $\nabla_{y, \max }$ are the maximum value of the $x$ - and $y$-component image gradients, and $L_{\max , \text { global }}$ and $L_{\text {min,global }}$ are set to 1 and 0 , the maximum and minimum values of the brain likelihood map. In practice, $L_{\min , \text { global }}$ can be larger than 0 to bias the particle to track tighter on the brain boundary, and this value was set to 0.3. Once the predicted measurement is defined, a measurement, $z_{\mathrm{k}}$, at the particle location is taken.

$$
z_{k}=\left[\begin{array}{llll}
\nabla_{x, k} & \nabla_{y, k} & L_{\max , k} & L_{\min , k}
\end{array}\right]^{T}
$$

where $\nabla_{x, k}$ and $\nabla_{y, k}$ are the $x$ - and $y$-components of the 2D gradient at the particle location, and $L_{\max , k}$ and $L_{\min , k}$ are the maximum and minimum values of the brain likelihood map in the neighborhood of the particle, defined by Eq. 1. Measurement is obtained by lookup of the gradient image and brain likelihood map at the particle locations.

Step 3: Each particle is assigned a weight by calculating $P\left(z^{*} \mid z\right)$, the probability of the predicted measurement occurring given the particle measurement, assuming a Gaussian model of uncertainty in gradient and position. ${ }^{12}$

$$
\begin{aligned}
& P\left(z^{*} \mid z\right)=\exp \left(-\frac{1}{2}\left(z^{*}-z\right) S^{-1}\left(z^{*}-z\right)\right) \\
& S=\left[\begin{array}{cccc}
\sigma_{\nabla, x}^{2} & 0 & 0 & 0 \\
0 & \sigma_{\nabla, y}^{2} & 0 & 0 \\
0 & 0 & \sigma_{\max , L}^{2} & 0 \\
0 & 0 & 0 & \sigma_{\min , L}^{2}
\end{array}\right]
\end{aligned}
$$

$S$ is the covariance matrix, where $\sigma_{\nabla, x}^{2}=\sigma_{\nabla, y}^{2}=\sigma_{\max , L}^{2}=\sigma_{\min , L}^{2}=$ $\sqrt{5^{*} 10^{-5}}$. These values were optimized experimentally. Additionally, if the particle is within 1 pixel of past boundary points (previous three boundary points in time not included), the particle weight is set to zero to avoid back-tracking.

Step 4: The particles are resampled according to their weights using a low variance sampling algorithm. ${ }^{29}$ In this resampling method, the particles of lowest weight are discarded and the particles of highest weight are duplicated. The low variance sampler has the advantage of not discarding any particles if they are all of equal weight.

To illustrate this algorithm, we represent each weighted particle as a bar with width proportional to its weight. The particle weights are normalized so the sum of all weights is equal to 1. Appendix Fig. $4 \mathrm{~A}$ shows an example of 5 particles with equal weight. A random number, $r$, is chosen between 0 and $\Pi^{-1}$ where $\Pi$ is the number of particles. In this example $\Pi^{-1}=0.2$. If the weight of particle $1, w_{1}$, is smaller than $r$, it is discarded. In this case, $w_{1}$ is larger than $r$ and it is placed in the set of new particles. $\Pi^{-1}$ is added to $r$ and we choose the next particle. If $w_{1}$ is greater than $r+\Pi^{-1}$ particle 1 is again added into the next set of particles. In this case, $w_{1}<r+\Pi^{-1}$. We then ask if the cumulative weight of particles 1 and $2, w_{1}+w_{2}$, is greater than $r+\Pi^{-1}$. In this case it is and particle 2 is added into the set of new particles. This process continues until we have an equal number of particles in the new set as were in the previous set. In Appendix Fig. $4 \mathrm{~A}$, all particles are of equal weight so none are discarded and none are duplicated. In Appendix Fig. 4B, however, the 5 particles are not weighted equally. If the same random number is chosen, we see that only particles 1,2 , and 4 move on to become the new set of particles, while particles 3 and 5 are discarded. Additionally, particles 2 and 4 are duplicated. It is possible, although less likely, for particles 3 or 5 to move on to the next set of particles if the random number were different. It remains possible (but unlikely) for particles of low weight to propagate to the resampled set. This is in fact desirable since they still represent a possible state. ${ }^{29}$ 

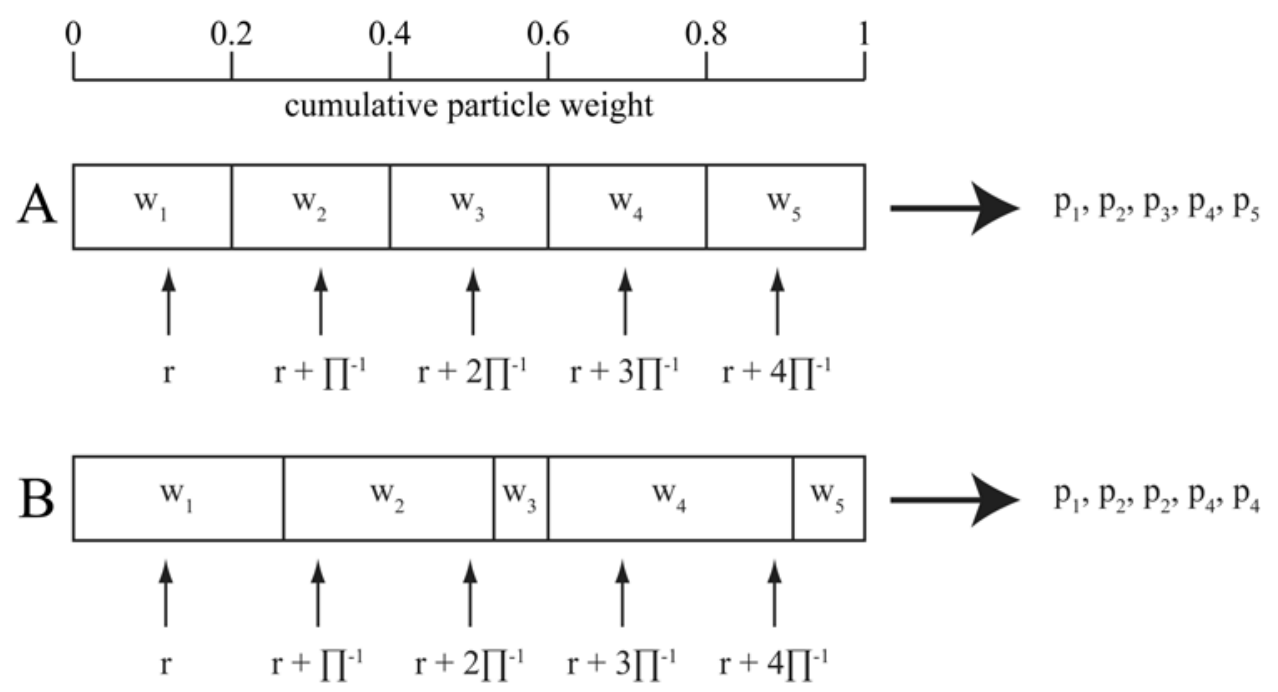

APPENDIX FIG. 4. The low variance sampling algorithm: the particles, $w_{n}$, are represented here by bars with their width corresponding to their weight. $\Pi^{-1}$ is added to a random number, $r, \Pi$ times, and the corresponding particle is placed into the set of new particles, $p_{i}$.

Resampling will always introduce bias into the distribution. Over time, this bias will reduce the size of the particle distribution as the filter reduces its measure of uncertainty. To avoid this process, the particles undergo roughening where extra Gaussian process noise, $u \sim N(0,0.5)$, is added to position and velocity after the particles have been resampled. ${ }^{27}$

Repeat Steps 1-4: The particle filter is applied recursively until the seed point is reached, forming a closed shape, and a binary mask is created from the closed brain boundary. Error checks are in place to stop the particle filter before a closed shape is formed if either the mean of the particle weights becomes too low or a particle travels off the image. If either of these errors occur, the particle filter restarts from the seed point, traveling in the opposite initial direction. If an error occurs a second time, the user is prompted to enter a new seed point.

The covariance matrix was able to change the behavior of the particle filter by changing the importance that was placed on the different measurements. $\sigma_{\nabla, x}^{2}$ and $\sigma_{\nabla, y}^{2}$ were kept equal to each other, as were $\sigma_{\max , L}^{2}$ and $\sigma_{\min , L}^{2}$. This was done to give equal importance to the $x$ and $y$ gradient and maximum and minimum values of the likelihood map neighborhood. The particle filter ran optimally when all of these values were equal.

The minimum expected tissue value, $L_{\text {min, slobal }}$, should be equal to zero. However, in practice, the particle cloud tended to track the brain tissue better and stay close to the boundary when this number was increased to 0.3. Any higher and too much tissue was lost outside of the segmented mask. Because this number was larger than zero, it was necessary to dilate the segmented brain mask to correct for missing tissue just outside of the mask. It also helped to have an erosion step following the dilation step that acted to fill in small holes in pixel classification..$^{28}$ The brain mask was optimized to have a larger dilation step while the fluid mask was optimized to have a larger erosion step. The fluid mask required a larger erosion step so as not to include the fluid outside the brain.

\section{Final Segmentation}

The final segmentation is created by multiplying the binary mask created in the edge-tracing step with the brain and fluid likelihood maps created in the classification step. This results in a soft segmentation where multiple tissue types can exist in a single pixel with different probabilities. ${ }^{3}$ The soft segmentation is thresholded to create a hard, or binary, final segmentation of brain and fluid.
For example, one voxel could be $70 \%$ brain and $30 \%$ fluid in a soft segmentation. The conversion to a hard segmentation would label this voxel as purely brain since it is more likely to contain more brain than fluid. The total volume is estimated by multiplying the voxel volume by number of voxels.

\section{User Interface}

Each slice of an image stack is segmented independently. Note that the user has the option to add as many boundaries as needed to completely segment the brain. Further, a post-processing step can be applied if the particle filter fails to create a closed shape, misses obvious tissue or segments tissue that is not brain. Here, we use a semi-automatic live-wire edge-tracing algorithm that snaps to nearby edges for this post-processing option. ${ }^{20}$

\section{Evaluation}

The particle filter segmentation tool was evaluated using a simulated T2-weighted MRI from the Montreal Neurological Institute BrainWeb. 6,7 These data sets are of a single brain, simulated with different levels of noise and RF inhomogeneity. The percent noise is the percent ratio of the standard deviation of the white Gaussian noise versus the signal for CSF. The RF inhomogeneity is represented by linearly scaled intensity gradients added to the image. The data set has a 3 -mm slice thickness with a $1 \times 1-\mathrm{mm}$ in-plane resolution. The algorithm was optimized using two simulations, the first without noise or intensity non-uniformity, and the second with $5 \%$ noise and $40 \%$ non-uniformity. Parameters optimized included the calculation of intensity thresholds, covariance matrix, the predicted measurements, as well as the option to dilate and erode the segmented mask (Appendix Table 1). ${ }^{28}$ Binary dilation allows the mask to expand by changing any pixel of value 0 to 1 if it is connected to a pixel of value 1 . Similarly, binary erosion shrinks the mask by changing any pixel of value 1 to 0 if it is connected to a pixel of value $0 .^{28}$ As each parameter was adjusted, the Dice overlap coefficient, $D(A, B)$, was measured, where $A$ is the segmented region and $B$ is the region of the ground truth. ${ }^{10,34}$ The parameters were individually adjusted until the Dice coefficient was maximized for the segmentation of the 2 simulations.

$$
D(A, B)=\frac{2 A \cap B}{A+B}
$$


APPENDIX TABLE 1. Values of optimized parameters for MRI segmentation*

\begin{tabular}{ll}
\hline \multicolumn{1}{c}{ Parameter } & Value \\
\hline Brain threshold & 0.616 \\
\hline Brain mask dilation & 7 \\
\hline Brain mask erosion & 4 \\
\hline Fluid threshold & 0.345 \\
\hline Fluid mask dilation & 2 \\
\hline Fluid mask erosion & 8 \\
\hline
\end{tabular}

* Brain and fluid thresholds take values between 0 and 1 . Dilation and erosion values are measured in the size of a $2 \mathrm{D}$ square pixel mask.

where $\cap$ indicates intersection. $D(A, B)$ is 1 for perfect overlap, and 0 for no overlap.

After optimization, the algorithm was tested with simulations of $0 \%-9 \%$ noise and $0 \%-40 \%$ intensity nonuniformity. The segmentation for brain and fluid was compared with the ground truth using the segmented volume and the Dice overlap measure.

\section{Adaptation for CT Images}

This method was adapted for use with CT DICOM images to demonstrate the versatility of the particle filter algorithm. As there is no ground truth for CT data, we optimized the algorithm using one CT data set that was segmented manually using AMIRA 5.2 (Visage Imaging). AMIRA was used to assign each pixel in the image as brain, fluid, or neither. This allows us to optimize the algorithm with the same method that was used for MRI data. The tissue classification thresholds, covariance matrix, minimum expected tissue value, thresholds to convert the soft brain and fluid segmentations to hard segmentations, and the number of pixels to dilate and erode the final brain and fluid masks were all adjusted to obtain the largest Dice overlap measure (Appendix Table 2)..$^{10,34}$

We then segmented 5 CT data sets of pediatric hydrocephalus with the particle filter and, for validation, by hand using the Cavalieri method with Stereo Investigator (MicroBrightField Sciences). The Cavalieri method uses a randomly assigned grid with intermarker distance of $5 \mathrm{~mm}$ overlain on the CT images. Markers
APPENDIX TABLE 2. Values of optimized parameters for CT segmentation

\begin{tabular}{ll}
\hline \multicolumn{1}{c}{ Parameter } & Value \\
\hline Brain threshold & 0.833 \\
\hline Brain mask dilation & 4 \\
\hline Brain mask erosion & 2 \\
\hline Fluid threshold & 0.867 \\
\hline Fluid mask dilation & 1 \\
\hline Fluid mask erosion & 10 \\
\hline
\end{tabular}

within the region of interest were assigned to either brain or fluid. The Pearson product-moment correlation coefficient, $r$, was used to measure the linear correlation between measurements with the particle filter and Cavalieri methods. It is calculated as the estimate of the covariance of the 2 variables divided by the product of the variances. ${ }^{12}$ This is followed by a 1-tailed t-test to measure the significance of the correlation. We have archived data samples and code for the particle filter, which are available for download as a Code Archive here. Links to online video clips demonstrating the particle filter tracking the edge of the brain on CT and MR images are provided within the text.

For CT images, the threshold between air and fluid, $t_{1}$, was calculated as the minimum value of fluid selected minus $2 \%(0.02)$. The threshold between brain and fluid, $t_{2}$, was calculated as the mean of the maximum value of fluid selected and the minimum value of brain selected. The threshold between brain and skull was calculated as the maximum value of brain selected plus $2 \%(0.02)$. The elements of the covariance matrix $S$ were defined as: $\sigma_{\nabla, x}^{2}=\sigma_{\nabla, y}^{2}$ $=\sqrt{10^{-4}}$ and $\sigma_{\max , L}^{2}=\sigma_{\min , L}^{2}=\sqrt{5^{*} 10^{-4}}$. The minimum and maximum expected tissue values, $L_{\text {max,global }}$ and $L_{\text {min,global }}$ from Eq. 5 , were set to 1 and 0.5 for $\mathrm{CT}$, which became the maximum and minimum values of the brain likelihood map. This means that we no longer expect a pixel containing tissue at the edge of the brain to have a value between 1 and 0 (where 1 is white and 0 is black). Setting the minimum expected tissue value to 0.5 serves to bias the particle cloud to follow the brain more tightly and reduces the chances of the cloud veering off track. 